\title{
Comparison of The Accuracy of Clinicians' Prediction of Survival and Palliative Prognostic Score: An East Asian Cross-Cultural Study
}

Yusuke Hiratsuka

Tohoku Daigaku https://orcid.org/0000-0003-2334-3893

\section{Seok-Joon Yoon}

Chungnam National University Hospital

Sang-Yeon Suh ( $\sim$ lisasuhmd@hotmail.com )

Dongguk University

\section{Sung-Eun Choi}

Dongguk University

\section{David Hui}

The University of Texas MD Anderson Cancer Center

\section{Sun-Hyun Kim}

Catholic Kwandong University International Saint Mary's Hospital

\section{Eon Sook Lee}

Inje University Ilsan Paik Hospital

\section{Sun Wook Hwang}

Korea University

\section{Shao-Yi Cheng}

National Taiwan University

Ping-Jen Chen

Kaohsiung Medical University

Masanori Mori

Seirei Mikatahara Byoin

Takashi Yamaguchi

Konan Byoin

\section{Tatsuya Morita}

Seirei Mikatahara Byoin

\section{Satoru Tsuneto}

Kyoto University: Kyoto Daigaku

\section{Akira Inoue}

Tohoku University School of Medicine: Tohoku Daigaku Daigakuin Igakukei Kenkyuka Igakubu 


\section{Research Article}

Keywords: Clinicians' prediction of survival, Palliative Prognostic Score, Prognostication, Advanced cancer, East Asia

Posted Date: August 9th, 2021

DOl: https://doi.org/10.21203/rs.3.rs-680169/v1

License: (c) (i) This work is licensed under a Creative Commons Attribution 4.0 International License. Read Full License

Version of Record: A version of this preprint was published at Supportive Care in Cancer on November 6th, 2021. See the published version at https://doi.org/10.1007/s00520-021-06673-0. 


\section{Abstract}

\section{Purpose:}

No study has been conducted to compare the clinicians' prediction of survival (CPS) with Palliative Prognostic Scores (PaP) across countries. We aimed to compare the performance of the CPS in PaP (PaP-CPS), the PaP without the CPS, and the PaP total scores in patients with advanced cancer in three East Asian countries.

\section{Methods:}

We compared the discriminative accuracy of the three predictive models (the PaP-CPS [the score of the categorical CPS of PaP], the PaP without the CPS [sum of the scores of only the objective variables of $\mathrm{PaP}$, and the PaP total score) in patients in Japan, Korea, and Taiwan. We calculated the area under the receiver operating characteristic curve (AUROC) for 30-day survival to compare the discriminative accuracy of these three models.

\section{Results:}

We analyzed 2,072 patients from three countries. The AUROC for the PaP total scores was 0.84 in patients in Japan, 0.76 in Korea, and 0.79 in Taiwan. The AUROC of the PaP-CPS was 0.82 in patients in Japan, 0.75 in Korea, and 0.78 in Taiwan. The AUROC of the PaP without the CPS was 0.75 in patients in Japan, 0.66 in Korea, and 0.67 in Taiwan.

\section{Conclusion:}

The PaP total scores and the PaP-CPS consistently showed similar discriminative accuracy in predicting 30-day survival in patients in Japan, Korea, and Taiwan. It may be sufficient for experienced clinicians to use the CPS alone for estimating the short-term survival (less than one month) of patients with faradvanced cancer. The PaP may help to improve prognostic confidence and further reduce subjective variations.

\section{Introduction}

The ability of clinicians to predict survival time is vital for palliative patients and their families. End-of-life care based on prognostication can be important to an individual patient's benefit [1], and the clinicians' prediction of survival (CPS) is a classical tool used for this purpose. However, the CPS is known to be far more optimistic than actual survival [1-5]. A previous study demonstrated that the accuracy of the CPS varied between $23 \%$ and $78 \%$ [6]. And the CPS could be affected by the patient-clinician relationship even in the case of expert clinicians [7]. Therefore, clinicians are recommended to use the CPS with objective prognostic indicators $[1,8,9]$. 
The Palliative Prognostic Score (PaP) is comprised of the CPS, Karnofsky Performance Status (KPS), two symptoms, and two laboratory values from the complete blood count [10]. The PaP aims to predict 30day survival and has been validated in various clinical settings [11-18]. Thus, the European Association for Palliative Care recommended PaP as an evidence-based prognostic tool [9]. However, inexperienced clinicians may hesitate to approach the PaP because it can be difficult for them to formulate the CPS [1921].

A few studies have investigated whether the CPS enhanced the accuracy of prognostic models [22, 23]. The performance of the CPS and the PaP can be influenced by each country's environment including the medical systems and the educational background of the clinicians [6]. In addition, the clinician-patient relationship in each country may affect the CPS performance [24]. To the best of our knowledge, no study has been conducted to compare CPS with the accuracy of prognostic models in patients in different countries. We thought that investigating diverse populations would be confirmative since previous studies showed contradictory results $[20,25]$. Therefore, we aimed to compare the performance of the PaP-CPS (the score of the categorical CPS of PaP), the PaP without the CPS, and the PaP total scores to predict the 30-day survival of patients in three East Asian countries.

\section{Methods}

\section{Participants}

This was a secondary analysis of an international multicenter prospective cohort study called the East Asian collaborative cross-cultural Study to Elucidate the Dying Process (EASED). It aimed to investigate the dying process and the end-of-life care of inpatients with far-advanced cancer in palliative care units (PCUs) nationwide in Japan, South Korea, and Taiwan. We consecutively enrolled eligible, newly admitted inpatients in the participating PCUs during the study period. All observations were performed within the range of routine clinical practice. The inclusion criteria were (1) adults aged $\geq 18$ years old in Japan and Korea and $\geq 20$ years old in Taiwan, (2) patients with locally extensive or metastatic cancer, and (3) patients newly admitted to a participating PCU. The exclusion criteria were (1) a scheduled discharge within one week and (2) refusal of the patient or her/his family to enroll in the study.

\section{Data collection}

The palliative clinicians evaluated the patients' symptoms by direct observation at admission. We followed up with the discharged patients for six months after admission to the PCUs in Japan and Taiwan, and six months after discharge from the PCUs in Korea. We defined survival time as mortality in and outside hospitals calculated by subtracting the admission date from the death date. We collected data on the CPS, KPS, dyspnea, anorexia, leukocyte counts, and lymphocyte percentages to calculate the $\mathrm{PaP}[10]$.

\section{Data analysis and statistics}


In this study, the variables were defined as 1) PaP-CPS, which was the score of the categorical CPS of the $\mathrm{PaP}$, and was selected by the clinicians (range $0-8.5$ points);

2) $\mathrm{PaP}$ without $\mathrm{CPS}$, which was the combined score of the five clinical and laboratory variables in the $\mathrm{PaP}$, and was equivalent to the PaP total score minus the PaP-CPS (range $0-9$ points); and 3 ) the $\mathrm{PaP}$ total score, which was the PaP-CPS + PaP without the CPS (range $0-17.5$ points).

We generated a receiver operating characteristic (ROC) curve using the true positive rate and the false positive rate. An ROC curve examines the performance of a binary outcome (e.g., alive or dead at 30 days). We calculated the area under the ROC curve (AUROC) to compare the discriminative accuracy of the PaP total score, the PaP-CPS, and the PaP without the CPS for the 30 -day survival predictions of the patients in three countries.

All analyses were performed using IBM Statistical Package for Social Science (SPSS) Statistics for Windows, version 26.0 (IBM Corp., Armonk, NY, USA). A P-value below 0.05 was considered statistically significant.

\section{Ethics}

In accordance with the ethical guidelines for human research of Japan's Ministry of Health, Labor, and Welfare, the requirement for informed patient consent was waived in Japan because of the observational nature of the study. In Korea and Taiwan, informed consent was obtained from the patients or their families (if the patient lacked the capacity to make decisions). This study obtained approval from the local Institutional Review Boards of all participating institutions. The Independent Ethics Committee of the Tohoku University School of Medicine (approval no. 2016-1-689) also approved this study.

\section{Results}

\section{Patient characteristics}

The data of 2,638 patients from 37 PCUs (22 in Japan, 11 in Korea, and four in Taiwan) were available. The patients were enrolled from January 2017 to September 2018. We excluded 564 patients (474 in Japan, 15 in Korea, and 75 in Taiwan) because laboratory data or CPS were missing to calculate the PaP. Among the eligible 2,074 patients, two Japanese patients were excluded because of missing data on survival time. Thus, 2,072 patients (1,422 in Japan, 320 in Korea, and 330 in Taiwan) were analyzed, which included 1,078 men (726 in Japan (51.1\%), 174 in Korea (54.4\%), and 178 in Taiwan (53.9\%)). The median survival time was 19.0 days (95\% confidence interval [Cl]: $17.3-20.7$ ) in Japan, 23.0 days (95\% Cl: 20.2 - 25.8) in Korea, and 15.0 days (95\% Cl: 12.6 - 17.5) in Taiwan. The characteristics of the patients are shown in Table 1.

\section{Clinician characteristics}


Table 2 shows the characteristics of the 162 clinicians (69 in Japan, 29 in Korea, and 64 in Taiwan) participating in this study. The study included 92 male clinicians (52 in Japan (75.4\%), eight in Korea (27.6\%), and 32 in Taiwan (50.0\%)). According to the countries, their clinical backgrounds such as specialty, clinical experience, and clinical experience with palliative care were diverse. The most common specialty of the Japanese clinicians was palliative care, whereas it was family medicine for those in Korea and Taiwan. The clinical careers and palliative care careers of the Taiwanese clinicians were a little bit shorter than those of the Japanese or Korean clinicians because some Taiwanese resident clinicians participated in about half of the institutions in this study. There was no difference in the number of faradvanced cancer patients treated by the clinicians of the three countries in one year.

\section{Accuracy of the PaP total score, PaP-CPS, and PaP without the CPS for estimating 30-day survival}

Table 3 shows the accuracy of the PaP total score, PaP-CPS, and PaP without the CPS for estimating 30day survival. The AUROC of the PaP total scores was 0.84 (95\% Cl: $0.82-0.86)$ in Japan, $0.76(95 \% \mathrm{Cl}$ : $0.70-0.81)$ in Korea, and 0.79 ( $95 \% \mathrm{Cl}: 0.74-0.84)$ in Taiwan. The AUROC of the PaP-CPS was 0.82 (95\% Cl: 0.80 - 0.84) in Japan, 0.75 (95\% Cl: 0.70 - 0.81) in Korea, and 0.78 (95\% Cl: $0.72-0.83)$ in Taiwan. The AUROC of the PaP without the CPS was 0.75 (95\% Cl: $0.73-0.78)$ in Japan, 0.66 (95\% Cl: $0.60-0.72)$ in Korea, and 0.67 (95\% Cl: $0.61-0.74)$ in Taiwan.

\section{PaP total score vs. PaP-CPS}

The comparison of the PaP total scores and PaP-CPS results is shown in Table 3 and Figure 1. The AUROCs of the PaP total scores in the patients in all three countries were greater than those of the PaPCPS but were not statistically significant. The phenomenon was common between the three countries.

\section{PaP total score vs. PaP without the CPS}

The AUROCs of the PaP total scores in all three countries were greater than those of the PaP without the CPS and were statistically significant (Table 3 , Figure 1 ).

\section{PaP-CPS vs. PaP without the CPS}

The AUROCs of the PaP-CPS in all three countries were also significantly greater than that of the PaP without the CPS (Table 3, Figure 1).

\section{Discussion}

The aim of this study was to compare the performance of the PaP-CPS, the PaP without the CPS, and the $\mathrm{PaP}$ total score to predict 30-day survival in patients in three East Asian countries. Our study demonstrated that the PaP total score and the PaP-CPS alone consistently showed similar discriminative accuracy in predicting 30-day survival in patients in all three East Asian countries. 
The study findings showed that the CPS alone was almost as accurate as the PaP total score in predicting 30-day survival in patients in Japan, Korea, and Taiwan. A previous study showed a similar accuracy of the CPS and the PaP in predicting 30-day, 14-day, and 7-day survival [23]. However, the previous study was a single-center study and thus, further extended studies are needed. The strengths of the current study were that we included a large number of participants from three different East Asian countries. And consistently, the accuracy of the CPS was comparable to that of the PaP total score in the patients in all three countries. We assume our results are explained in terms of patients' and clinicians' factors. As for patient factors, our study population included advanced cancer patients with a short median survival time (19 days in Japan, 23 days in Korea, and 15 days in Taiwan). The CPS could be relatively accurate in patients with more predictable trajectories and experienced clinicians [26]. Therefore, the patients in the three countries studied may have a more predictable clinical course than those in the recent studies. Consistently, the accuracy of the CPS may be higher in patients with shorter predicted survivals [27]. Regarding the clinician factors, we assume that the participating clinicians were experts in predicting survival. Experts are known to have smaller errors in prognostic predictions and could predict more accurate prognoses $[5,24]$. The accuracy of the CPS is influenced by clinician-related factors such as the lack of knowledge on the survival of patients under palliative care, the duration of the clinician-patient relationship, and training on prognostication [24]. In Japan, all palliative care clinicians are required to be educated through a comprehensive educational program provided by the Japanese Society for Palliative Medicine $[28,29]$. The PaP-CPS in Japan showed higher accuracy than that of the other two countries. Although the difference was not statistically significant, it can be explained by the following. In Japan, the National Cancer Control Act established in 2007 has actively promoted palliative care for cancer patients immediately after their diagnosis to improve distress, symptom burden, and quality of life. It enables palliative care physicians to manage patients with cancer continuously from an earlier phase of the disease, allowing time to observe the changes in a patient. In Korea, standardized educational programs are mandatory for all palliative clinicians to start their careers in the palliative care field. They also should complete at least two educational programs by the Korean Society of Hospice and Palliative Care per year. In Taiwan, a continuous educational program sponsored by the Taiwan Academy of Hospice Palliative Medicine has been provided for clinicians for palliative care similar to that in Japan. Additionally, although some participating clinicians were resident clinicians in half of the institutions, all of them received supervision from attending physicians in terms of CPS evaluation. The palliative care clinicians in all three countries generally would have provided information to families regarding survival predictions, [30] since children regard talking about death to their elderly parents contrary to 'filial piety' under the Confucian culture [31]. Our findings showed that the CPS was formulated accurately while palliative clinicians were cognizant of indirect communication with the patients.

This study had several limitations. First, this study used laboratory data to calculate the PaP within one week before or three days after study entry when available. However, if the timing of laboratory data collection was extended or shortened, the PaP total score might be different. Second, the median survival of our patients was relatively short at $15-23$ days. Because patient survival time is an important factor influencing the accuracy of the CPS, we could not guarantee that the accuracy of our CPS findings would 
be similar in patients with longer survival times. Third, this study population was in PCUs and may not represent the general population of patients with far-advanced cancer. The CPS can differ according to patients' life expectancies and their places of care. Moreover, the CPS might be formulated by experts demonstrating excellent prognostic performance. Lastly, our findings may be influenced by the accessibility to palliative care services and PCU admission in each country.

\section{Conclusion}

The PaP total score and the PaP-CPS showed similar discriminative accuracy in consistently predicting 30-day survival in patients in Japan, Korea, and Taiwan. It may be sufficient for experienced clinicians to use the CPS alone for estimating the short-term survival (less than one month) of patients with faradvanced cancer. The PaP may help to improve prognostic confidence and further reduce subjective variations.

\section{Declarations}

\section{Acknowledgments:}

We appreciate the EASED investigators in Japan, Korea and Taiwan for sincere data collection of this study.

We are grateful to Harrisco Encorrection (Seoul, South Korea) for proofreading this manuscript for grammar and clarity.

\section{Funding}

This work was supported in part by a Grant-in-Aid from the Japanese Hospice Palliative Care Foundation; Grant Numbers $16 \mathrm{H} 05212$ and 16KT0007.

\section{Conflicts of interest}

The authors declare that there is no conflict of interest.

\section{Data availability}

The data that support the findings of this study are available from the corresponding author, Sang-Yeon Suh, upon reasonable request. All authors agree to provide data to the journal for review if needed.

\section{Code availability}

Not applicable.

\section{Author contributions}


Yusuke Hiratsuka: Conceptualization, Data curation, Investigation, Methodology, Project administration, Writing - original draft, Writing - review and editing

Seok-Joon Yoon: Conceptualization, Formal analysis, Investigation, Methodology, Project administration, Writing - original draft, Writing - review and editing

Sang-Yeon Suh: Conceptualization, Investigation, Methodology, Project implementation, Supervision, Writing - review and editing

Sung Eun Choi: Formal analysis, Writing - review and editing

David Hui: Supervision, Writing - review and editing

Sun-Hyun Kim: Investigation, Writing - review and editing

Eon Sook Lee: Investigation, Writing - review and editing

Sun Wook Hwang: Investigation, Writing - review and editing

Shao-Yi Cheng: Investigation, Writing - review and editing

Ping-Jen Chen: Investigation, Writing - review and editing

Masanori Mori: Conceptualization, Investigation, Methodology, Project administration, Resources, Supervision, Writing - review and editing

Takashi Yamaguchi: Investigation, Writing - review and editing

Tatsuya Morita: Funding acquisition, Investigation, Writing - review and editing

Satoru Tsuneto: Supervision, Writing - review and editing

Akira Inoue: Investigation, Supervision, Writing - review and editing

\section{Ethics approval}

This study obtained was approved by the local Institutional Review Boards of all participating institutions. The independent ethics committee of Tohoku University School of Medicine (approval no. 2016-1-689) approved this study.

\section{Consent to participate}

In accordance with the ethical guidelines for human research of the Ministry of Health, Labor, and Welfare in Japan, patients' informed consent was waived in Japan because of the completely observational nature of the study. Patients were provided the opportunity to opt out.In South Korea and Taiwan, 
informed consent was obtained from the patients or their families (in case the patient lacked the capacity to decide).

\section{Consent for publication}

The authors affirm that human research participants provided informed consent for publication of the article.

\section{References}

1. Hui D (2015) Prognostication of survival in patients with advanced cancer: predicting the unpredictable. Cancer Control 22:489-497

2. Selby D, Chakraborty A, Lilien T et al (2011) Clinician accuracy when estimating survival duration: the role of the patient's performance status and time based prognostic categories. J Pain Symptom Manage 42:578-588

3. Amano K, Maeda I, Shimoyama S et al (2015) The accuracy of physicians' clinical predictions of survival in patients with advanced cancer. J Pain Symptom Manage 50:139-146

4. Cheon S, Agarwal A, Popovic M et al (2016) The accuracy of clinicians' predictions of survival in advanced cancer: a review. Ann Palliat Med 5:22-29

5. Tavares T, Oliveira M, Gonc alves J et al (2018) Predicting prognosis in patients with advanced cancer: a prospective study. Palliat Med 32:413-416

6. White N, Reid F, Harris A et al (2016) A systematic review of predictions of survival in palliative care: how accurate are clinicians and who are the experts? PLoS One 11:e0161407

7. Christakis NA (1999) Prognostication and bioethics. Daedalus 128:197-214

8. Chow E, Harth T, Hruby G et al (2001) How accurate are physicians' clinical predictions of survival and the available prognostic tools in estimating survival times in terminally ill cancer patients? A systematic review. Clin Oncol 13:209-218

9. Maltoni M, Caraceni A, Brunelli C et al (2005) Prognostic factors in advanced cancer patients: evidence based clinical recommendations - a study by the Steering Committee of the European Association for Palliative Care. J Clin Oncol 23:6240-6248

10. Pirovano M, Maltoni M, Nanni $O$ et al (1999) A new palliative prognostic score: a first step for the staging of terminally ill cancer patients. J Pain Symptom Manage 17:231-239

11. Glare P, Virik K (2001) Independent prospective validation of the PaP score in terminally ill patients referred to a hospital-based palliative medicine consultation service. J Pain Symptom Manage 22:891-898

12. Glare PA, Eychmueller S, McMahon P (2004) Diagnostic accuracy of the palliative prognostic score in hospitalized patients with advanced cancer. J Clin Oncol 22:4823-4828

13. Tassinari D, Montanari L, Maltoni $M$ et al (2008) The palliative prognostic score and survival in patients with advanced solid tumors receiving chemotherapy. Support Care Cancer 16:359-370 
14. Naylor C, Cerqueira L, Costa-Paiva LH et al (2010) Survival of women with cancer in palliative care: use of the palliative prognostic score in a population of Brazilian women. J Pain Symptom Manage 39:69-75

15. Kurashima AY, Latorre Mdo R, Camargo B (2010) A palliative prognostic score for terminally ill children and adolescents with cancer. Pediatr Blood Cancer 55:1167-1171

16. Tarumi Y, Watanabe SM, Lau F et al (2011) Evaluation of the Palliative Prognostic Score (PaP) and routinely collected clinical data in prognostication of survival for patients referred to a palliative care consultation service in an acute care hospital. J Pain Symptom Manage 42:419-431

17. Numico G, Occelli M, Russi EG et al (2011) Survival prediction and frequency of anticancer treatment in cancer patients hospitalized due to acute conditions. Role of clinical parameters and PaP score. Support Care Cancer 19:1823-1830

18. Baba M, Maeda I, Morita T et al (2015) Survival prediction for advanced cancer patients in the real world: a comparison of the palliative prognostic score, delirium-palliative prognostic score, palliative prognostic index and modified prognosis in palliative care study predictor model. Eur $\mathrm{J}$ Cancer 51:1618-1629

19. Suh SY, Choi YS, Shim JY et al (2010) Construction of a new, objective prognostic score for terminally ill cancer patients: a multicenter study. Support Care Cancer 18:151-157

20. Hui D, Park M, Liu D et al (2016) Clinician prediction of survival versus the Palliative Prognostic Score: which approach is more accurate? Eur J Cancer 64:89-95

21. Yoon SJ, Suh SY, Hui D et al (2021) Accuracy of the Palliative Prognostic Score With or Without Clinicians' Prediction of Survival in Patients With Far Advanced Cancer. J Pain Symptom Manage 61:1180-1187

22. Farinholt P, Park M, Guo $Y$ et al (2018) A comparison of the accuracy of clinician prediction of survival versus the palliative prognostic index. J Pain Symptom Manage 55:792-797

23. Hui D, Ross J, Park M et al (2020) Predicting survival in patients with advanced cancer in the last weeks of life: How accurate are prognostic models compared to clinicians'. estimates? Palliat Med 34:126-133

24. Christakis NA, Lamont EB (2000) Extent and determinants of error in doctors' prognoses in terminally ill patients: prospective cohort study. BMJ 320:469-472

25. Yoon SJ, Jung JG, Kim JS et al (2014) Comparison of accuracy among prognostic scores for predicting life expectancy in Korean patients with cancer with weeks of survival. Am J Hosp Palliat Care 31:845-852

26. White N, Reid F, Vickerstaff $V$ et al (2020) Specialist palliative medicine physicians and nurses accuracy at predicting imminent death (within 72 hours): a short report. BMJ Support Palliat Care $10: 209-212$

27. Hui D, Ross J, Park M et al (2019) Predicting survival in patients with advanced cancer in the last weeks of life: how accurate are prognostic models compared to clinicians'. estimates? Palliat Med $34: 126-133$ 
28. Nakazawa $Y$, Yamamoto $R$, Kato $M$ et al (2018) Improved knowledge of and difficulties in palliative care among physicians during 2008 and 2015 in Japan: Association with a nationwide palliative care education program. Cancer 124:626-635

29. Yamamoto R, Kizawa Y, Nakazawa Y et al (2015) Outcome evaluation of the Palliative care Emphasis program on symptom management and Assessment for Continuous Medical Education: nationwide physician education project for primary palliative care in Japan. J Palliat Med 18:45-49

30. Yamaguchi T, Maeda I, Hatano Y et al (2021) Communication and behavior of palliative care physicians of cancer patients near end-of-life in three East Asian countries. J Pain Symptom Manage 61:315-322

31. Bedford O, Yeh KH (2019) The History and the Future of the Psychology of Filial Piety: Chinese Norms to Contextualized Personality Construct. Front Psychol 10:100

\section{Tables}


Table 1

General Characteristics of the Subjects $(n=2,072)$

\begin{tabular}{|c|c|c|c|}
\hline & $\begin{array}{l}\text { Japan } \\
1,422)\end{array}$ & $\begin{array}{l}\text { Korea }(n= \\
320)\end{array}$ & $\begin{array}{l}\text { Taiwan }(n= \\
330)\end{array}$ \\
\hline Age (years) & $72.7 \pm 12.3$ & $68.0 \pm 12.3$ & $67.5 \pm 14.0$ \\
\hline Sex (male) & $726(51.1)$ & $174(54.4)$ & $178(53.9)$ \\
\hline \multicolumn{4}{|l|}{ Primary cancer site } \\
\hline Lung & $249(17.5)$ & $46(14.4)$ & $66(20.0)$ \\
\hline Gastroesophageal & $196(13.8)$ & $45(14.1)$ & $23(7.0)$ \\
\hline Colorectal/small intestine & $194(13.7)$ & $50(15.6)$ & $45(13.6)$ \\
\hline Hepatobiliary/pancreas & $267(18.8)$ & $91(28.4)$ & $78(23.6)$ \\
\hline Breast & $97(6.8)$ & $18(5.6)$ & $16(4.8)$ \\
\hline Gynecological & $84(5.9)$ & $14(4.4)$ & $12(3.6)$ \\
\hline Urological & $100(7.0)$ & $16(5.0)$ & $23(7.0)$ \\
\hline Head and neck & $58(4.1)$ & $8(2.5)$ & $34(10.3)$ \\
\hline \multicolumn{4}{|l|}{ (excluding thyroid) } \\
\hline Others & $177(12.4)$ & $32(10.0)$ & $33(10.0)$ \\
\hline \multicolumn{4}{|l|}{ KPS in PaP score categories } \\
\hline$\geq 30$ (0 point) & $1180(83.0)$ & $272(85.0)$ & $200(60.6)$ \\
\hline 10-20 (2.5 points) & $242(17.0)$ & $48(15.0)$ & $130(39.4)$ \\
\hline $\begin{array}{l}\text { Dyspnea in PaP score categories (present, } 1 \\
\text { point) }\end{array}$ & $560(39.4)$ & $121(37.8)$ & $186(56.4)$ \\
\hline $\begin{array}{l}\text { Anorexia in PaP score categories (present, } 1.5 \\
\text { points) }\end{array}$ & $1163(81.8)$ & $257(80.3)$ & $234(70.9)$ \\
\hline \multicolumn{4}{|l|}{$\begin{array}{l}\text { Leukocyte count in PaP score categories (cells } \\
\text { per } \mathrm{mm}^{3} \text { ) }\end{array}$} \\
\hline$<8,500$ (0 point) & $655(46.1)$ & $160(50.0)$ & $97(29.4)$ \\
\hline $8,500-10,999$ (0.5 point) & $280(19.7)$ & $53(16.6)$ & $63(19.1)$ \\
\hline$>11,000$ (1.5 points) & $487(34.2)$ & 107 (33.4) & $170(51.5)$ \\
\hline \multicolumn{4}{|c|}{$\begin{array}{l}\text { Abbreviation: KPS, Karnofsky performance status; PaP, palliative prognostic score; CPS, clinicians' } \\
\text { prediction of survival; PaP-CPS, score of the categorical CPS of the PaP }\end{array}$} \\
\hline
\end{tabular}




\begin{tabular}{|c|c|c|c|}
\hline & $\begin{array}{l}\text { Japan } \\
1,422)\end{array}$ & $\begin{array}{l}\text { Korea }(n= \\
320)\end{array}$ & $\begin{array}{l}\text { Taiwan }(n= \\
\text { 330) }\end{array}$ \\
\hline \multicolumn{4}{|c|}{ Lymphocyte percentage in $\mathrm{PaP}$ score categories } \\
\hline$\geq 20 \%$ (0 point) & $159(11.2)$ & $55(17.2)$ & $30(9.1)$ \\
\hline $12-19.9 \%$ (1.0 point) & $350(24.6)$ & $94(29.4)$ & $55(16.7)$ \\
\hline$<12 \%$ (2.5 points) & $913(64.2)$ & $171(53.4)$ & $245(74.2)$ \\
\hline \multicolumn{4}{|c|}{ CPS (weeks, categorical CPS of PaP) } \\
\hline$>12$ (0 point) & $112(7.9)$ & $42(13.1)$ & $21(6.4)$ \\
\hline 11-12 (2 points) & $7(0.5)$ & $2(0.6)$ & $1(0.3)$ \\
\hline 7-10 (2.5 points) & $242(17.0)$ & $87(27.2)$ & $52(15.8)$ \\
\hline 5-6 (4.5 points) & $353(24.8)$ & $76(23.7)$ & $69(20.9)$ \\
\hline $3-4$ (6 points) & $233(16.4)$ & $64(20.1)$ & $51(15.4)$ \\
\hline$\leq 2$ (8.5 points) & $475(33.4)$ & $49(15.3)$ & $136(41.2)$ \\
\hline PaP total score (range $0-17.5$ ) & $9.88(4.21)$ & $8.43(3.91)$ & $11.27(3.91)$ \\
\hline PaP-CPS (range 0-8.5) & $5.37(2.69)$ & $4.26(2.58)$ & $5.77(2.70)$ \\
\hline $\mathrm{PaP}$ without CPS (range $0-9.0$ ) & $4.51(2.08)$ & $4.17(2.04)$ & $5.50(1.99)$ \\
\hline Survival time (days) & $\begin{array}{l}19.0(17.3- \\
20.7)\end{array}$ & $\begin{array}{l}23.0(20.2- \\
25.8)\end{array}$ & $\begin{array}{l}15.0(12.6- \\
17.5)\end{array}$ \\
\hline \multicolumn{4}{|c|}{$\begin{array}{l}\text { Abbreviation: KPS, Karnofsky performance status; PaP, palliative prognostic score; CPS, clinicians' } \\
\text { prediction of survival; PaP-CPS, score of the categorical CPS of the PaP }\end{array}$} \\
\hline \multicolumn{4}{|c|}{$\begin{array}{l}\text { Footnote: The data are expressed as the mean (standard deviation) or } \mathrm{n}(\%) \text { or median value }(95 \% \\
\text { confidence interval). }\end{array}$} \\
\hline
\end{tabular}


Table 2

Characteristics of the Participating Clinicians in 3 Countries $(n=162)$

\begin{tabular}{|c|c|c|c|}
\hline & $\begin{array}{l}\text { Japan }(n= \\
69)\end{array}$ & $\begin{array}{l}\text { Korea }(n= \\
29)\end{array}$ & $\begin{array}{l}\text { Taiwan }(n= \\
64)\end{array}$ \\
\hline Sex (male) & $52(75.4)$ & $8(27.6)$ & $32(50.0)$ \\
\hline \multicolumn{4}{|l|}{ Specialty } \\
\hline Internal medicine & $10(14.5)$ & $5(17.2)$ & $1(1.6)$ \\
\hline Surgery & $1(1.4)$ & $0(0.0)$ & $0(0.0)$ \\
\hline Palliative care & $51(73.9)$ & $0(0.0)$ & $18(28.1)$ \\
\hline Family medicine & $3(4.3)$ & $22(75.9)$ & $51(79.7)$ \\
\hline Others & $4(5.8)$ & $2(6.9)$ & $11(17.2)$ \\
\hline Clinical experiences (years) & $11.8 \pm 6.5$ & $12.7 \pm 7.8$ & $5.8 \pm 3.5$ \\
\hline $\begin{array}{l}\text { Clinical experiences of } \\
\text { palliative care (years) }\end{array}$ & $5.5 \pm 5.0$ & $6.8 \pm 5.5$ & $2.8 \pm 3.1$ \\
\hline $\begin{array}{l}\text { Number of patients with far-advanced cancer treated } \\
\text { in a year }\end{array}$ & $98.8 \pm 92.5$ & $\begin{array}{l}129.3 \pm \\
151.9\end{array}$ & $\begin{array}{l}111.1 \pm \\
141.3\end{array}$ \\
\hline
\end{tabular}

Table 3

Comparison of 30-day Survival Predictions by PaP Total Score, PaP-CPS, and PaP without CPS

$$
\text { Japan }(n=1,422) \quad \text { Korea }(n=320) \quad \text { Taiwan }(n=330)
$$

AUROC for 30-day survival $(95 \% \mathrm{Cl})$

$\begin{array}{llll}\text { PaP total score } & 0.84(0.82-0.86)^{\mathrm{a}} & 0.76(0.70-0.81)^{\mathrm{a}} & 0.79(0.74-0.84)^{\mathrm{a}} \\ \text { PaP-CPS } & 0.82(0.80-0.84)^{\mathrm{a}} & 0.75(0.70-0.81)^{\mathrm{a}} & 0.78(0.72-0.83)^{\mathrm{a}}\end{array}$

\begin{tabular}{|c|c|c|}
\hline $\mathrm{PaP}$ without $\mathrm{CPS}$ & $0.75(073-0.78)^{b}$ & $0.66(0.60-0.72)^{b}$ \\
\hline
\end{tabular}

Abbreviation: AUROC, area under the receiver operating characteristic curve; $\mathrm{Cl}$, confidence interval; $\mathrm{CPS}$, clinicians' prediction of survival; PaP, palliative prognostic score; PaP-CPS, score of the categorical CPS of $\mathrm{PaP}$

Footnote: Superscript a is significantly higher than superscript $b$. The three different predictive models were compared within each of the three subject groups.

\section{Figures}



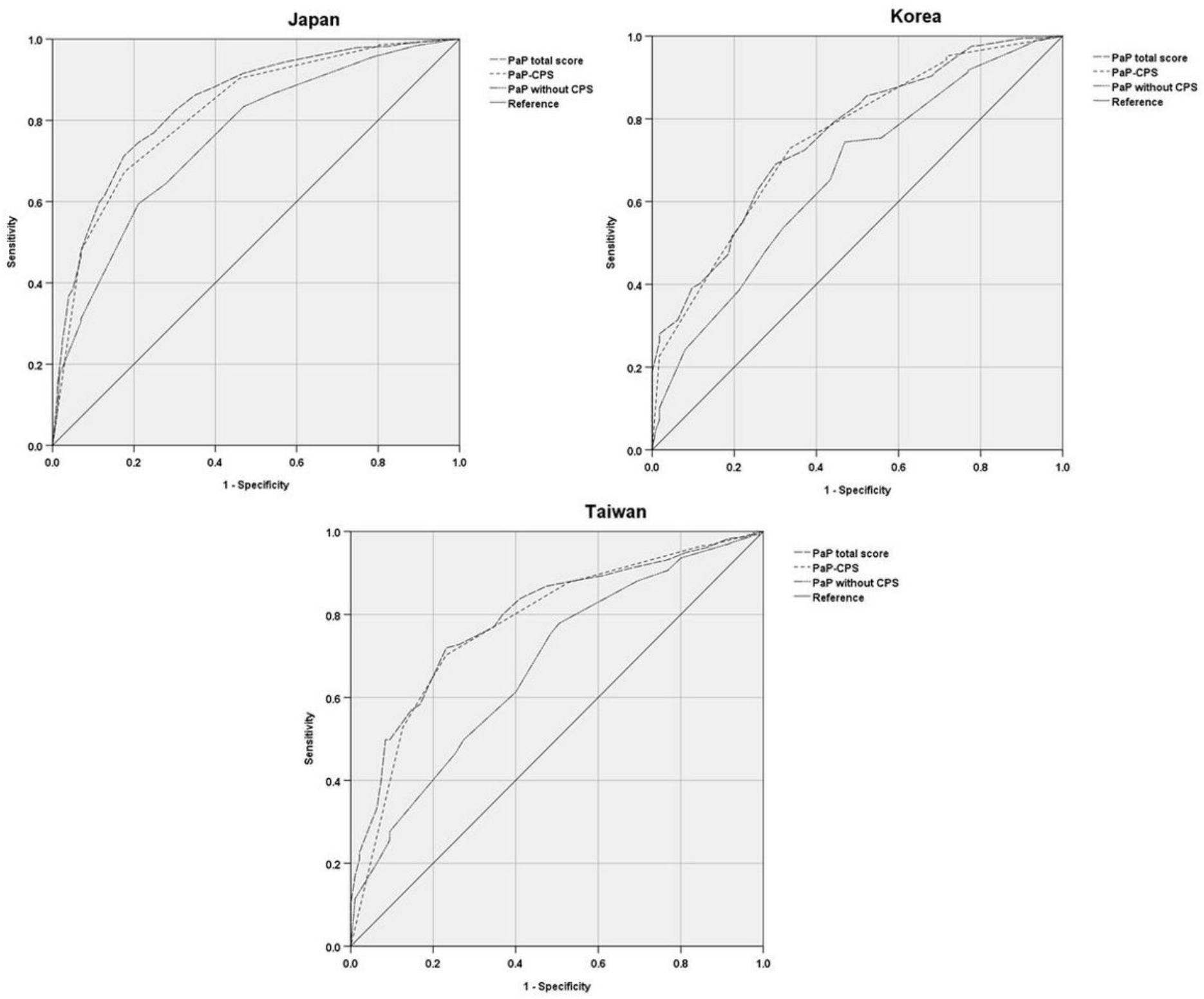

Figure 1

Receiver Operating Curves of the PaP Total Score, PaP without the CPS, and PaP-CPS in 3 Countries Abbreviation: CPS, clinicians' prediction of survival; PaP, palliative prognostic score; PaP-CPS, score of the categorical CPS of PaP 